\title{
ÜBERSEERECHT UND NEOKOLONIALISMUS
}

\author{
Von BRUn-OTtTo BRYDE
}

Im Augustheft 1970 der von der Deutschen Akademie für Staats- und Rechtswissenschaft „Walter Ulbricht“ in Potsdam-Babelsberg herausgegebenen Zeitschrift „Staat und Recht" setzen sich Professor Gerhard Brehme und Klaus Hutschenreuter, beide von der Sektion Afrika- und Nahostwissenschaften der Leipziger Karl-MarxUniversität, kritisch mit dem westdeutschen Beitrag zur Erforschung von Staat und Recht der Entwicklungsländer auseinander ${ }^{1}$. Daß die Überseespezialisten der DDR von den Bemühungen ihrer Kollegen im Westen Kenntnis nehmen, ist zu begrüßen, und es ist des Nachdenkens wert, daß die Beschäftigung westdeutscher Juristen mit den Entwicklungsländern in der DDR mehr Aufmerksamkeit zu finden scheint als bei den Mitjuristen in der Bundesrepublik. Das mag daran liegen, daß die Rechtslehre der Bundesrepublik sich dieses Themas erst spät angenommen hat ${ }^{2}$. Brehme und Hutschenreuter geben eine knappe Darstellung der Geschichte dieses Forschungszweiges ${ }^{3}$, die im wesentlichen zutreffend ist. Beleuchtet wird vor allem der Zusammenhang zwischen Kolonialmachtstellung und wissenschaftlichem Interesse, der dazu führte, daß man sich außerhalb der Kolonialmetropolen erst mit dem Unabhängigkeitsprozeß stärker für die Gebiete Afrikas und Asiens zu interessieren begann.

Als Hauptvertreter der staats- und rechtswissenschaftlichen Beschäftigung mit den Entwicklungsländern unter den westdeutschen Juristen sehen die Autoren ${ }^{4}$ Krüger $^{5}$, Ronneberger ${ }^{6}$ und Herrfahrdt ${ }^{7}$ an. Mit diesen setzen sie sich eingehend auseinander und lassen sie auch selbst in ausführlichen Zitaten zu Wort kommen. Allerdings ist die Darstellung teilweise veraltet ${ }^{8}$. Auch sonst leidet sie an mangelnden Informationen über die tatsächliche Lage dieses Wissenszweiges in Westdeutschland. Eine rasche Bedeutungszunahme juristischer Entwicklungsländerforschung ${ }^{9}$ ist (leider) kaum zu erwarten. Auch die Vorstellung, es würden Zentren gebildet ${ }^{10}$ und Nachwuchskader gefördert ${ }^{11}$ kann bei demjenigen nur ein wehmütiges Lächeln hervorrufen, der um die Schwierigkeiten weiß, gerade für dieses Gebiet die erforderlichen sachlichen und personellen Mittel einzuwerben ${ }^{12}$. Es läßt sich nur schwer sagen, ob für diese Vorstellung von einem planmäßigen und zentralisierten Ausbau der juristischen Entwicklungsländerforschung nur Informationsmängel verantwort-

1 G. Brehme/K. Hutschenreuter, Zur Rolle der westdeutschen Staats- und Rechtswissenschaft im System des Neokolonialismus, in: Staat und Recht 1970 (H. 8), S. 1254 ff.

2 Die Feststellung der beiden Autoren, „daß sich die juristische Wissenschaft in Westdeutschland bis zum Beginn der 60er Jahre nicht systematisch mit den Veränderungen in Staat und Recht der Entwicklungsländer beschäftigte" (a. a. O., S. 1256), kann man nur unterstreichen.

3 a. a. O., S. $1255 \mathrm{ff}$.

4 a. a. O., S. 1257.

5. Vgl. H. Krüger, Grundzüge der Verfassungsbildung in den neu entstandenen Staaten. Deutsche Landesreferate zum VI. Internationalen Kongreß für Rechtsvergleichung in Hamburg 1962, Berlin und Tübingen 1962, S. 309 ff.; ders. Das Programm - Verfassung und Recht in Übersee, in: VRU 1968, S. 3 ff.

$6 \mathrm{Vgl}$. Ronneberger, Das Verfassungsproblem in den Entwicklungsländern, in: Der Staat 1962, S. $39 \mathrm{ff}$.

7 Vgl. Herrfahrdt, Staatsgestaltungsfragen in den Entwicklungsländern Asiens und Afrikas, Karlsruhe 1965. Aus jüngerer Zeit wäre auf die von den Autoren nicht verwertete letzte Arbeit Heinrich Herrfahrdts vor seinem Tode zu verweisen: Nationalstaat und Uberschneidungsräume in Europa und in den Entwicklungsländern, in: VRƯ 1970, S. $1 \mathrm{ff}$.

8 Die Nachweise a. a. O., S. 1258 f., zeigen, daß die Autoren im wesentlichen nur Literatur bis 1968 verwertet haben.

9 a. a. O., S. 1258 .

10 a. a. O., S. 1258 .

11 a. a. O., S. 1259 .

12 Weder den "Imperialisten ${ }^{\star}$ in Bonn noch den "Kapitalisten ${ }^{*}$ an Rhein und Ruhr ist offensichtlich klar, welche bedeutende Hilfsfunktion die Rechtswissenschaft im "System des Neokolonialismus spielen könnte. 
lich sind oder auch eine gerade im Ost-West-Verhältnis leicht auftretende Tendenz, hinter allen Erscheinungen im gegnerischen Lager einen ordnenden politischen Willen zu vermuten.

Die Neigung, jeder noch so sachlichen Aussage westdeutscher Wissenschaftler eine böse Absicht $\mathrm{zu}$ unterstellen, durchzieht jedenfalls den ganzen Artikel ${ }^{13}$. So wird zwar deren Bemühen konstatiert, durch interdisziplinäre Zusammenarbeit und moderne Forschungsmethoden $\mathrm{zu}$ genaueren und komplexeren Ergebnissen zu kommen, aber dieses Bemühen beruht natürlich nur auf dem Verlangen, zu „für die praktische Politik des westdeutschen Imperialismus nützlichen Ergebnissen und Aussagen $\mathrm{zu}$ gelangen"14. Wobei nur zu fragen bleibt, wer einen Vorteil davon hätte, wenn sie ungenaue und vereinfachende Aussagen machten. Der Auffassung Krügers, Souveränität schließe die Freiheit der Wahl der Verfassung ein und wegen dieser Wahl stehe Dritten weder das Recht der Intervention noch der Zensur $\mathbf{z u}^{15}$, stimmen Brehme und Hutschenreuter ausdrücklich $\mathrm{zu}$, vermuten aber sofort, die Wahl des sozialistischen Weges wolle der westdeutsche Autor den Entwicklungsländern damit keinesfalls freistellen ${ }^{16}$. Woher wissen sie das? Ahnlich argwöhnen sie, Krüger wolle mit der Befürwortung staatlicher Wirtschaftsplanung in den Entwicklungsländern ${ }^{17}$ die „kapitalistische Planifikation“ empfehlen ${ }^{18}$. Auch das ist eine unbelegte Unterstellung. Sehr viel näher liegt die Vermutung, daß weder östliche noch westliche Planungssysteme, die beide für Industriestaaten entwickelt worden sind, den Bedürfnissen von Entwicklungsländern gerecht werden.

Prokapitalistische und imperialistische Absichten sehen die Autoren insbesondere darin, daß man den Begriff der Modernisierung zum Ausgangspunkt der Entwicklungsländerforschung wählt ${ }^{19}$. Dabei gehen sie allzu global davon aus, daß Modernität als Entwicklungsziel die Empfehlung der Übernahme westlicher Verfassungsmodelle beinhaltet ${ }^{20}$. Daß die von den Kolonialherren übernommenen Verfassungsordnungen in den meisten Ländern sehr schnell scheiterten, übersieht auch im Westen niemand, obwohl dazu angemerkt werden muß, daß es bemerkenswerte Ausnahmen gibt (Indien) und daß z. B. Ghana, nachdem es mit einem stark personalisierten Einparteiensystem unter Nkrumah und einer Militärdiktatur typische Formen afrikanischer Verfassungswirklichkeit durchlaufen hat, $1969 \mathrm{zu}$ einer Verfassung nach dem Westminster-Modell zurückgekehrt ist. (Dabei ist die Zukunft dieser Verfassungsmodelle natürlich ungewiß.)

Die Alternative, den Begriff der Modernisierung nicht auf eine Entwicklung zu westlichen, sondern zu „nördlichen“ Gesellschaftsformen zu beziehen, wobei offenbleibt, ob sich die Entwicklungsländer für einen östlichen, westlichen oder dritten Weg entscheiden, muß den Autoren aus der DDR als „entwicklungsländerspezifische Variante der Konvergenztheorie "21 noch verdächtiger sein. Nun ist die Konvergenztheorie nicht nur im Osten umstritten ${ }^{22}$. Man braucht ihr aber nicht

\footnotetext{
13 Dazu vgl. die Leitartikel von Chung-hua Jih-pao, 21. Nov. 1970, und Kao-hsiung Hsin-wen-pao, ten wird (a. a. O., S. 1270).

14 a. a. O., S. 1259.

15 Krüger, Programm, S. 7.

16 a. a. O., S. 1267.

17 Vgl. Krüger, Programm, S. 28.

18 a. a. O., S. 1268.

19 a. a. O., S. $1260 \mathrm{ff}$.

20 a. a. O., S. 1262. Die dort zitierten Außerungen Ronnebergers sind jedoch nicht typisch. Aus Krügers Ausführungen (vgl. z. B. Programm, a. a. O., S. 7) läßt sich leicht entnehmen, daß er den Entwidklungsländern keinen bestimmten Verfassungstyp aufdrängen will. Herrfahrdt lehnt eine Orientierun der Entwicklungsländer am demokratischen Nationalstaat westlicher Prägung ausdrücklich ab (vgl. VRƯ 1970, S. 1 ff., S. 5).

21 a. a. O., S. 1261 .

21 Zur westlichen Kritik vgl. u. a. K.' P. Hensel, Annäherung der Wirtschaftssysteme? in: Deutsche Studien 1969 , S. $225 \mathrm{ff}$.
} 
anzuhängen, um feststellen zu können, daß die Unterschiede aus der Perspektive der Entwicklungsländer angesichts des aus deren Sicht ungleich fundamentaleren Gegensatzes von Norden und Süden verschwimmen. Politische Etikette ändern nichts daran, daß ihrer sozial-ökonomischen Struktur nach Bundesrepublik und DDR mehr gemeinsam haben als DDR und die Volksrepublik Kongo (Brazzaville) oder die Bundesrepublik und die Elfenbeinküste. Auf diese Weise in die Nähe der Kapitalisten gerückt zu werden, kann Marxisten nicht sympathisch sein, aber Interessenidentität läßt sich eben nicht nur zwischen Entwicklungsländern und westlichen Industriestaaten ${ }^{23}$, sondern auch $\mathrm{zwischen} \mathrm{Entwicklungsländern} \mathrm{und}$ östlichen Industriestaaten nicht herstellen. Das ist auf internationaler Ebene, sobald es nicht um Deklamationen, sondern um handfeste ökonomische Interessen geht, deutlich zu erkennen ${ }^{24}$.

Damit soll den DDR-Autoren nicht verwehrt werden, eine Differenzierung in kapitalistische und nicht-kapitalistische Staaten $\mathrm{zu}$ machen und diese für wichtiger zu halten ${ }^{25}$. Ob sie allerdings sehr sinnvoll und der tatsächlichen Lage der Länder der Dritten Welt angemessen ist, muß bezweifelt werden. Man hat allzu of t den Eindruck, daß die Einteilung dieser Länder in kapitalistisch-reaktionäre und fortschrittliche mehr nach ihrer außenpolitischen Orientierung als nach der tatsächlichen inneren Situation erfolgt. Das ist nicht verwunderlich, wenn die Autoren selbst zugestehen, daß die Klassendifferenzierung in vielen betreffenden Staaten gering ist ${ }^{26}$. $\mathrm{Da}$ andererseits auch sie nicht übersehen, daß der Begriff „modern“ für einige Entwicklungsziele einen Sinn hat ${ }^{27}$, fragt sich, ob der Begriff der Modernisierung nicht gerade, wenn man eine eurozentrische Betrachtung vermeiden und vom Selbstverständnis der Länder Afrikas und Asiens ausgehen will, einen fruchtbaren Ansatzpunkt bietet, als die Einordnung in die Schemata der Alten Welt ${ }^{28}$.

Aber auch wenn man nicht bereits in dem Forschungsansatz neokolonialistische Zielsetzungen entdeckt, bleibt der Vorwurf des Neokolonialismus bei jeder Beschäftigung mit den Entwicklungsländern ernst zu nehmen. Eine Stellungnahme zu diesem Vorwurf erfordert eine Prüfung der Gründe, aus denen man sich mit Verfassung und Recht der Entwicklungsländer beschäftigt und daran anschließend die Frage, ob die Intentionen oder auch nur das Ergebnis der auf Grund ihrer vorgenommenen Forschungen dem Neokolonialismus dienen.

Für die Wahl der Entwicklungsländer als Gegenstand rechtswissenschaftlicher Forschung können sehr verschiedene Motivationen ausschlaggebend sein. Welche Herausforderung der Prozeß der Modernisierung für die Wissenschaft bedeutet und welche Aufgaben er ihr stellt, hat Krüger in dem programmatischen Aufsatz für diese Zeitschrift nachgewiesen ${ }^{29}$. Mit welchem gespannten Interesse die Staatslehre die jungen Staaten beobachten wird, ist leicht einzusehen, wenn man bedenkt,

23 So Brehme / Hutschenreuter, a. a. O., S. 1270.

24 Eine klare Konfrontation zwischen Industriestaaten und Entwicklungsländern gibt es z. B. in der Meeresbodenfrage. So erfolgte die Abstimmung über die Resolution 2574 D (XXIV) v. 15.12 .1969 (UN Doc. A/RES 2574 [XXIV] v. 15. 1. 70) eindeutig nach dem Kriterium: entwickelt gegen unterentwickelt. Vgl. auch Kehden, Die Vereinten Nationen und die Nutzung des Bodens und Untergrundes des Hohen Meeres außerhalb der Grenzen nationaler Hoheitsgewalt, VRƯ 1969, S. 131 ff., S. 151. Auch in der UNCTAD wird ein Nord-Süd-Gegensatz deutlich, nachdem die Entwicklungsländer von den osteuropäischen Ländern mehr als allgemeine Willenserklärungen verlangen, vgl. Gosovic, UNCTAD: NorthSouth Encounter, International Conciliation (Mai 1968) Nr. 568, S. 26 f.

25 a. a. O., S. 1262.

26 a. a. O., S. 1262

27 a. a. O., S. 1263.

28 Diese Kritik muß sich natürlich auch gegen westliche Konzepte wie die eines „Entwicklungslandes im verfassungstheoretischen $\operatorname{Sinn}^{\alpha}$ (vgl. Ronneberger, a. a. O., S. 54 f.) oder einer nunvollendeten Demokratie ${ }^{\alpha}$ (Scupin) richten.

29 VRU 1968, S. 3 ff. 
daß der Vorgang des Entstehens eines Staates, der durch Jahrhunderte die Gedanken der Staatslehrer beschäftigt hat, hier beobachtbar und Phänomene wie das der Grenze $^{30}$ oder die Entwicklung von Staatsbewußtsein empirisch erforschbar werden.

Der Wert von Forschungen, die auf keiner anderen Motivation als wissenschaftlichem Sachinteresse beruhen, darf nicht unterschätzt werden ${ }^{31}$. Aber regelmäßig erwartet die Gesellschaft vom Wissenschaftler doch den Nachweis des praktischen Nutzens seiner Tätigkeit. Dieser Nachweis ist für unseren Forschungsbereich nicht schwer zu führen. $\mathrm{Zu}$ Unrecht wird die Feststellung, daß auch die Industriestaaten aus den rechtlichen Erfahrungen der Entwicklungsländer lernen können, als bloße Geste $\operatorname{abgetan}^{32}$. Es gibt z. B. keinen Grund, warum Konstruktionen in überseeischen Gesetzen europäischen Ländern nicht als Vorbild dienen sollten ${ }^{33}$. Das wäre nicht erstaunlich, sondern es ist im Gegenteil wahrscheinlich, daß neue Normierungen in jungen Staaten, die auf den Erfahrungen der europäischen Rechtswissenschaft aufbauen können, ohne durch gesetzgeberische Traditionen beengt $\mathrm{zu}$ sein, von höherem Niveau sind als ihre europäischen Vorbilder ${ }^{34}$. Dem könnte entgegengehalten werden, daß solche Gesetze oft unter maßgeblichem Einfluß europäischer oder amerikanischer Juristen entstanden sind. Aber auch dann beruhen sie auf dem politischen Willen des Gesetzgebers, und es bleibt festzuhalten, daß z. B. René David in Äthiopien Rechtsreformen durchsetzen konnte, deren Verwirklichung in Frankreich nicht möglich war $^{35}$. Der Wert von Forschungen über Entwicklungsländerrecht erschöpft sich jedoch nicht in der Möglichkeit, Rechtsfiguren in verbesserter Gestalt zurückzurezipieren. Sehr viel wichtiger sind die Erkenntnisse über die Funktion des Rechts unter verschiedenen wirtschaftlichen und sozialen Bedingungen, die ein solches Studium verspricht. Insbesondere für Möglichkeiten, Recht zur Gesellschaftsgestaltung einzusetzen, bieten die Entwicklungsländer mehr Anschauungsmaterial als die Staaten der Alten Welt, in denen das Recht der wirtschaftlichen und sozialen Entwicklung gewöhnlich nachfolgt. Unmittelbar praktische Relevanz hat die Überseerechtsforschung schließlich auf den Rechtsgebieten, deren Kenntnis für die zunehmenden Kontakte mit diesen Ländern notwendig ist, wie z. B. Niederlassungs-, Arbeits- und Steuerrecht. Dieser Bereich ist natürlich neokolonialistischer Zielsetzungen besonders verdächtigi6. Wenn Brehme und Hutschenreuter allerdings auch das Recht der direkten menschlichen Kontakte (Familienrecht und Staatsangehörigkeit) in die Reihe schon wegen des Gegenstandes neokolonialistischer Forschungsgebiete stellen ${ }^{37}$, so fordern sie eigentlich einige sarkastische Bemerkungen über die Gründe heraus, wegen der diese Rechtsgebiete für westliche Staaten und ihre Bürger größere Relevanz haben als für die osteuropäischen Länder.

30 Vgl. H. Krüger, Allgemenne Staatslehre, 2. A. Stuttgart 1964, S. $20 \mathrm{ff}$.

31 Das folgt schon daraus, daß sich gerade nicht zweckgerichtete theoretische Forschung immer wieder als Arsenal praktisch verwertbarer Erkenntnisse erwiesen hat. Vgl. Lompe, Wissenschaftliche Beratung der Politik Göttingen 1966, S. 12

32 So Brehme / Hutschenreuter, a. a. O., S. 1269.

33 Beispiel könnte etwa die Normierung öffentlich-rechtlicher Verträge im äthiopischen Zivilgesetzbuch sein (Art. 3131-3306 Civil Code v. 1960).

34 Voraussetzung ist allerdings, daß man diese Chance nutzt und nicht hastig und mehr oder weniger unreflektiert global das Recht der ehemaligen Kolonialherren übernimmt wie z. B. in Somalia, vgl. Contini, The Somali Republic, London 1969, S. 45 f. Dazu die Besprechung in VRƯ 1970, S. $421 \mathrm{f}$

35 Vgl. David, Le code civil éthiopien de 1960, Rabels Z 1961, S. 668 ff.

36 Brehmer/ Hutschenreuter, a. a. O., S. 1259.

37 Ebendort. 
Auch rechtswissenschaftliche Beschäftigung mit der Dritten Welt kann schließlich auf das Motiv zurückgehen, „Entwicklungshilfe“ zu leisten. Daß zur Überwindung der Diskrepanz zwischen reichen und armen Ländern letztere nicht nur wirtschaftlicher und technischer Hilfe bedürfen, sondern auch der Vermittlung von Kenntnissen und Erfahrungen über das Funktionieren von staatlichen Einrichtungen und Recht, kann kaum bestritten werden. Solche Vermittlung kann durch Berater und Lehrer geschehen, die in die überseeischen Länder gehen, oder aber auch durch wissenschaftliche Forschung über die Adäquanz von Rechts- und Verfassungsmodellen für diese Länder und deren Publikation. Solche Aktivitäten westlicher Juristen müssen naturgemäß dem Verdacht begegnen, es sei eine Beeinflussung im kapitalistischen Sinne angestrebt ${ }^{38}$.

Juristische Entwicklungsländerforschung kann also auf Grund sehr verschiedener Motivationen betrieben werden. Unter welchen Bedingungen ihr der Vorwurf neokolonialistischer Zielsetzung gemacht werden kann, läßt sich erst entscheiden, wenn der Begriff des Neokolonialismus präzisiert ist. Brehme und Hutschenreuter verstehen als Neokolonialismus offensichtlich jeden westlichen Kontakt mit Entwicklungsländern ${ }^{39}$. Diese Verwendung des Begriffs hat sicher propagandistischen, keinesfalls aber wissenschaftlichen Wert. Das gilt gerade auch aus der Sicht der Entwicklungsländer, für die Kooperation auch mit dem Westen von erheblicher Bedeutung ist, woraus sich die Notwendigkeit ergibt, erwünschte ausländische Betätigungen von solchen $\mathrm{zu}$ unterscheiden, die als "neokolonialistische" abgelehnt werden. Der sinnvollste Weg zu einer Begriffsbestimmung ist es, sich an der Wortbedeutung zu orientieren: Neokolonialismus ist eine neue Form des Kolonialismus bzw. die Fortsetzung des Kolonialismus mit anderen Mitteln. Wenn man Kolonialismus als Negierung des Selbstbestimmungsrechts der Völker definieren kann, dann bedeutet Neokolonialismus, daß diese Selbstbestimmung, nachdem sie juristischformal errungen ist, materiell mit wirtschaftlichen, politischen oder militärischen Mitteln beeinträchtigt wird.

Diese Definition bedeutet allerdings nicht nur, daß westliche Betätigung in den Ländern der Dritten Welt nicht per se neokolonialistisch ist, sondern nur, wenn sie den Tatbestand der Beeinträchtigung der Selbstbestimmung erfüllt, sondern auch, daß östliche Tätigkeit in den Entwicklungsländern sich am gleichen Maßstab prüfen lassen muß. Eine solche Begriffsbestimmung macht es auch erst möglich, festzustellen, wann juristische Entwicklungsländerforschung „neokolonialistisch“ ist und wann nicht: neokolonialistisch ist sie dann, wenn sie sich in den Dienst von Bestrebungen stellt, die Selbstbestimmung der Entwicklungsländer zu beeinträchtigen.

Hier soll keine chronique scandaleuse neokolonialistischer Verfehlungen gegeben, aber auch nicht behauptet werden, daß solche Bestrebungen nicht bestehen ${ }^{40}$ und $\mathrm{da} ß$ es keine Juristen gibt, die sie unterstützen. Daß das nicht Zweck von Entwicklungsländerforschung sein darf, sollte nicht umstritten sein. Es dürfte aber auch schwerfallen, ernst zu nehmende westdeutsche Wissenschaftler zu finden, deren Arbeiten eine solche Intention zugrunde liegt. Man könnte eine derartige Ten-

$38 \mathrm{Vgl}$. Brehme / Hutschenreuter, a. a. O., S. $1269 \mathrm{f}$.

39 Das wird besonders a. a. O., S. 1259, deutlich, wo sie sogar dem Eherecht Relevanz für die praktische neokolonialistische Politik zusprechen.

40 Allerdings ist Vorsicht geboten: nicht jede enge Zusammenarbeit oder bloß faktisches Angewiesensein auf Hilfe bedeuten schon politische Abhängigkeit. Eine Abgrenzung zwischen bloß faktischer Abhängigkeit und deren Ausnützung ist ebenso schwierig wie notwendig. 
denz, sei sie bewußt oder unbewußt, höchstens daraus folgern, daß bestimmte Gebiete wie Niederlassungs-, Investitions-, Steuer- und Arbeitsrecht besondere Aufmerksamkeit finden ${ }^{41}$. Andererseits kann man nicht allein aus der an sich selbstverständlichen Tatsache, daß praktisch besonders relevante Fragen auch von der Wissenschaft aufgegriffen werden, schon auf unlautere Absichten schließen. Sogar eine überhaupt nicht praxisorientierte Wissenschaft müßte beachten, daß die wirtschaftliche Entwicklung zentrales Problem der Länder der Dritten Welt und damit das die Wirtschaft betreffende Recht natürlicher Forschungsschwerpunkt für ein juristisches Studium der Entwicklungsländer ist. Auch die Themenwahl kann also keine hinreichende Grundlage für den Vorwurf des Neokolonialismus sein, so daß der Nachweis, daß eine Aussage inhaltlich neokolonialistisch ist, notwendig bleibt.

Dieser Nachweis wird schwer sein, wenn es sich um rechtsvergleichende Arbeiten handelt, die sich darauf beschränken, den Rechtszustand des ausländischen Staats objektiv wiederzugeben ${ }^{42}$. Natürlich könnte man den Vorwurf erheben, auch mit der sachlichsten Darstellung würden dem potentiellen Ausbeuter wichtige Informationen gegeben. Wissenschaftliche Veröffentlichungen stehen jedem zur Verfügung. Arbeitsrechtliche Darstellungen können dem Arbeitgeber wie dem Arbeitnehmer Hinweise geben, Studien zum Niederlassungsrecht den ausländischen Investor wie die einheimischen Behörden auf Lücken aufmerksam zu machen. Der Wissenschaftler dürfte nicht mehr schreiben, wenn er verhindern wollte, daß die verschiedensten Personen mit den verschiedensten Interessen Gebrauch von seinen Forschungen machen. Soweit sie nur referiert, was in den Entwicklungsländern tatsächlich vorgeht, sich also um "Wahrheit" bemüht, kann der Überseerechtswissenschaft der Vorwurf des Neokolonialismus daher sinnvoll nicht gemacht werden.

Anderes könnte gelten, wenn Reformvorschläge gemacht oder Entwicklungsmodelle empfohlen werden. Da eine solche „juristische Entwicklungshilfe" von den Wertungen und Vorstellungen derer geprägt ist, die sie geben, könnte man hier den Versuch sehen, den Entwicklungsländern einen kapitalistischen Weg aufzudrängen. Allerdings ist die geistige Wirklichkeit im Westen differenzierter, als man es sich in der DDR vorstellt, und gerade Wissenschaftler, die sich in der Dritten Welt engagieren, werden in der Regel eher progressiv sein. Aber auch wenn sie Sozialisten sind, sehen sie kaum in den osteuropäischen Ländern eine besonders glückliche Verwirklichung des Sozialismus (eine Verwirklichung im übrigen, die sich schon wegen ihrer starken Bürokratisierung zur Übernahme durch die Entwicklungsländer allenfalls nach erheblichen Modifikationen eignet). Der Verdacht, die Entwicklungsländer sollen „kapitalistisch“ beeinflußt werden, könnte daher berechtigt sein, wenn der Begriff „kapitalistisch“ nur eine Leerformel für alle Gesellschaftsformen ist, die nicht die Zustimmung Moskaus finden. Neokolonialistisch wäre eine solche Beeinflussung jedoch nur, wenn sie die Freiheit der Entwicklungsländer, Staatsform und Entwicklungsweg selbst $\mathrm{zu}$ bestimmen, beeinträchtigte. Das ist bei wissenschaftlicher Einflußnahme, die sich auf keine anderen Machtmittel stützen kann als auf ihre Argumente, schon deshalb nicht der Fall, weil keine Gefahr besteht, daß sie einseitig erfolgt: die massive kommunistische Propaganda in den Entwicklungsländern schließt das aus. Diese empfiehlt schon

41 Vgl. Brehme / Hutschenreuter, a. a. O., S. 1259.

42 Vgl. auch Krüger, Programm, a. a. O., S. 7. 
deshalb sehr viel nachdrücklicher den eigenen Weg als den einzig richtigen, weil sie nicht die Skepsis vieler westlicher Wissenschaftler hinsichtlich der Übertragbarkeit des eigenen Verfassungsmodells teilt.

Informationen über westliches Verfassungs- und Rechtsdenken erfüllen daher die wichtige Funktion, die Entwicklungsländer mit der ganzen Fülle von Gestaltungsmöglichkeiten bekannt zu machen und sie so erst in den Stand zu versetzen, den eigenen Weg zu wählen, der selbstverständlich auch sozialistisch sein kann (und wahrscheinlich auch sein wird, wenn man diesen Begriff nicht für die Verhältnisse in den osteuropäischen Staaten reserviert). Solche Aktivitäten mögen daher unseren östlichen Nachbarn unangenehm sein, und es ist ihnen unbenommen, sie mit derogierenden Ausdrücken zu belegen. Sie neokolonialistisch zu nennen, bedeutet einen Mißbrauch der Sprache. 